\title{
Accompagnement dans l'apprentissage de l'argumentation par une équipe pluridisciplinaire : quels effets sur les acteurs?
}

Martine Paindorge, Virginie Lang, Virginie Libante, Pascal Reboul and Bertrand Aigle

\section{CpenEdition}

\section{Journals}

Electronic version

URL: http://journals.openedition.org/ripes/2717

DOI: 10.4000/ripes. 2717

ISSN: 2076-8427

Publisher

Association internationale de pédagogie universitaire

\section{Electronic reference}

Martine Paindorge, Virginie Lang, Virginie Libante, Pascal Reboul and Bertrand Aigle,

"Accompagnement dans l'apprentissage de l'argumentation par une équipe pluridisciplinaire : quels

effets sur les acteurs?", Revue internationale de pédagogie de l'enseignement supérieur [Online], 36(2) I

2020, Online since 23 November 2020, connection on 29 January 2021. URL: http://

journals.openedition.org/ripes/2717; DOI: https://doi.org/10.4000/ripes.2717

This text was automatically generated on 29 January 2021

Article L.111-1 du Code de la propriété intellectuelle. 


\title{
Accompagnement dans l'apprentissage de l'argumentation par une équipe pluridisciplinaire : quels effets sur les acteurs?
}

\author{
Martine Paindorge, Virginie Lang, Virginie Libante, Pascal Reboul and \\ Bertrand Aigle
}

\section{Introduction}

1 L'intention de cet article est de rendre compte de la réflexion de l'équipe pluridisciplinaire engagée dans les différentes phases d'une démarche d'innovation telle que décrite par Houart et al. (2011). Ainsi, après avoir conçu une Activité de Mise en Situation (AMS), puis l'avoir mise en œuvre et régulée depuis plusieurs années, il s'agit de se questionner sur les effets produits chez les acteurs avant d'envisager la pérennisation. Dans un premier temps, la recherche bibliographique permet de décrire et d'interroger le dispositif puis la méthodologie est définie pour recueillir les données. Enfin les résultats obtenus auprès des étudiants et de l'équipe pédagogique sont présentés et discutés.

\section{Un dispositif innovant}

2 La formation Cursus Master en Ingénierie (CMI) a vu le jour suite à l'appel à projet IDEFI 2011. (Initiatives d'Excellence en Formations Innovantes). Le CMI se caractérise notamment par la recherche comme outil de formation et par des pédagogies actives ( $25 \%$ du cursus) concrétisées par de nombreuses Activités de Mise en Situation (AMS) tout au long d'un cursus de cinq ans (apprentissage par résolution de problèmes, par projet, stages...). Ces AMS visent à rendre l'étudiant acteur de sa propre formation (Jacquet et al., 2014), par un contact étroit avec les laboratoires de recherche, les 
entreprises et une ouverture à l'international (semestres d'étude et/ou stages à l'étranger) ${ }^{1}$.

Ouvert dès 2012 à l'Université de Lorraine, le CMI Biologie-Santé-Environnement (CMI BSE) s'appuie sur la licence des Sciences de la Vie et sur deux masters : Microbiologie et Science du Vivant. Comme pour l'ensemble des CMI, la formation comprend des unités d'enseignement d'ouverture sociétale, économique et culturelle (OSEC) considérées comme indispensables pour former aux fonctions d'ingénieur. C'est dans ce cadre qu'est proposée dès le début de première année une AMS d'apprentissage de l'argumentation scientifique, définie ici comme «un acte de langage destiné à influencer un destinataire par la production d'énoncés oraux ou écrits (des arguments) qui soutiennent ou étayent une thèse, c'est-à-dire la conclusion à laquelle l'énonciateur cherche à faire adhérer son destinataire » (Chartrand et Elghazi, 2014, s.p.). Eu égard à la spécialité biologique de la formation, cette activité constitue une première rencontre des étudiants avec la littéracie scientifique, comprise ici comme l'ensemble des activités d'un chercheur qui impliquent l'usage de l'écriture, en réception et en production (définition adaptée de celle proposée par Jaffré, 2004, citée dans Delcambre et Lahanier-Reuter, 2012). Cette première AMS repose à la fois sur un travail des étudiants et sur leur accompagnement par une équipe pédagogique composée de spécialistes de différents domaines.

\section{Problématique}

4 L'accompagnement des étudiants par les intervenants est considéré comme un guidage pour aider l'accompagné à atteindre un but $^{2}$, principalement ici développer l'autonomie des étudiants dans la recherche et la lecture d'écrits scientifiques, dans une perspective de production d'arguments pour un débat. Le terme d'accompagnement est jugé polysémique par Cosnefroy et Annoot (2014). Pour autant, les deux auteurs, prenant appui sur les travaux d'autres chercheurs, mettent en évidence une convergence vers des caractéristiques communes. D'une part, ils mentionnent une finalité de réussite et d'autonomie des étudiants dans leur parcours, une relation quasi égalitaire entre apprenant et encadrant, ce dernier passant d'expert à personne ressource. D'autre part, l'accent est mis sur le changement de posture des encadrants : pédagogies actives, centrées sur l'étudiant, différentes du cours magistral, non plus basées sur une transmission de connaissances ou d'informations mais davantage sur une co-construction favorisant l'acquisition de compétences. La relation différente entre les différents personnels de l'Université partageant cette conception (enseignants, personnels de bibliothèque, personnel d'orientation) évolue également. Réussite et autonomie des étudiants et changement de posture des encadrants constituent les objectifs du dispositif AMS.

5 Après plusieurs années de mise en œuvre de cette AMS, il semble intéressant d'examiner la question: Quel(s) effet(s) de l'accompagnement pluridisciplinaire proposé à l'entrée du CMI pour les acteurs impliqués dans ce dispositif?

\subsection{L'accompagnement des étudiants}

6 Les statistiques ${ }^{3}$ du ministère de l'enseignement supérieur indiquent que seulement 57 \% environ des entrants de 2016-2017 en Licences Sciences de la Vie ont poursuivi 
l'année suivante. La situation n'est pas nouvelle et a donné lieu dès 2007 à la mise en place de dispositifs d'accompagnement dans le cadre du Plan Réussite en Licence (PRL) (Perret, 2014). La loi pour l'Orientation et la Réussite des Étudiants (ORE) de 2018 les maintient, cependant Garrau (2018) parle « d'accompagnement introuvable [...] faute de moyens humains et matériels». Ainsi, depuis une dizaine d'années, l'injonction institutionnelle vise à favoriser l'intégration et la réussite des étudiants, par des moyens divers.

7 Conçue pour répondre à ces finalités, l'AMS peut être décrite en utilisant les repères pour construire un dispositif d'accompagnement proposé par Rached (2017), i-e. le public visé, les fonctions du dispositif, le profil de l'accompagnateur, ses formes et modalités. Le public est constitué d'étudiants d'un bon niveau au lycée, volontaires pour s'engager dans une formation comprenant $20 \%$ d'heures supplémentaires d'enseignements en présentiel pour un même diplôme de Licence. Ils adhérent à la formation qu'ils suivent et n'ont pas besoin de consacrer à leurs apprentissages disciplinaires un temps augmenté par de la remédiation. En effet, ils ne font pas partie de la population des étudiants en difficulté au début de leur formation universitaire. Aussi la tâche demandée, préparer et présenter oralement un débat oral relatif à une controverse, comprise ici comme "un différend entre deux parties, mis en scène devant un public, tiers placé alors en position de juge " (Lemieux, 2007, p.195) leur permet de développer de nouvelles compétences. Les sujets sont choisis en relation avec les sciences, qui peuvent susciter des débats dans la société ou soulever des questions d'éthique. Les étudiants ont donc à approfondir leurs connaissances scientifiques sur les questions en jeu. Parmi les objectifs visés également, apprendre à rechercher et gérer l'information accessible via l'université ou en accès libre via le web semble essentiel pour la réussite des étudiants (Langevin, 2017). Cet apprentissage correspond aux compétences informationnelles, définies par l'utilisation de l'information de manière efficace et judicieuse, c'est-à-dire de savoir reconnaître son besoin d'information et de trouver, d'évaluer et d'utiliser cette information (American Library Association, 1989). Par ailleurs, la décision d'un travail réalisé en groupe est prise afin de fournir aux étudiants une expérience de partage du travail et de coconstruction de l'apprentissage résultant d'une interdépendance en obtenant une dynamique de groupe (Conefroy et Jézégou, 2013). De plus, cela favorise la construction d'un lien entre les étudiants d'un même groupe et l'esprit "promo » au sein d'une licence générale dès le début d'année universitaire.

8 La tâche confiée à chaque groupe nécessite ce que Gettliffe (2018) nomme un accompagnement dans l'acculturation (au sens d'adaptation) aux écrits universitaires. En effet, il est nécessaire d'accompagner l'accès aux écrits de recherche, la familiarisation aux écrits disciplinaires, l'entrée dans le processus d'argumentation destiné à produire et prendre appui sur un argumentaire. Le dispositif est pris en charge par une équipe pluridisciplinaire volontaire, composée de chercheurs et d'enseignants-chercheurs en biologie, d'une enseignante-chercheuse en sciences de l'éducation et d'une bibliothécaire. Chaque membre de l'équipe intervient individuellement, apportant ainsi sa propre contribution au cours de l'activité mais tous sont présents lors de la présentation orale finale. Le tableau suivant (Cf. Tableau 1) récapitule le fonctionnement de l'activité. Tous les acteurs sont indiqués, tant l'équipe pédagogique que les étudiants accompagnés, illustrant ainsi la nécessaire implication de chacun. 
Tableau 1. Présentation du fonctionnement de l'accompagnement pédagogique

\begin{tabular}{|c|c|c|c|c|c|}
\hline & \multicolumn{5}{|c|}{$\begin{array}{l}\text { Modalité de l'accompagnement pédagogique de } \\
\text { la découverte de largumentation scientifique }\end{array}$} \\
\hline Pour qui ? & \multicolumn{5}{|c|}{ Tous les étudiants de première année SV suivant le Cursus Master Ingéniérie (CMI) } \\
\hline Quand? & \multicolumn{5}{|c|}{ Premier semestre de licence sur une durée de 10 semaines } \\
\hline Par Qui ? & $\begin{array}{l}\text { Coordonnateur } \\
\text { (Enseignant- } \\
\text { chercheur) }\end{array}$ & Bibliothécaire & $\begin{array}{l}\text { Tuteurs (Enseignant- } \\
\text { chercheur, Chercheur) }\end{array}$ & $\begin{array}{l}\text { MCF Sciences de } \\
\text { l'éducation }\end{array}$ & Etudiant \\
\hline Sur quoi ? & $\begin{array}{l}\text { Présentation du } \\
\text { cahier des charges, } \\
\text { permettre le choix } \\
\text { des sujets par les } \\
\text { groupes }\end{array}$ & $\begin{array}{l}\text { Préparation de la } \\
\text { recherche d'information, } \\
\text { mise en forme de la } \\
\text { bibliographie, } \\
\text { sensibilisation au plagiat }\end{array}$ & $\begin{array}{l}\text { Accompagner les } \\
\text { étudiants dans le choix } \\
\text { des documents, } \\
\text { validation des arguments } \\
\text { scientifiques }\end{array}$ & $\begin{array}{l}\text { Préparation de } \\
\text { I'argumentation, mise } \\
\text { en forme du support, } \\
\text { préparation de la } \\
\text { communication orale }\end{array}$ & $\begin{array}{l}\text { Construction } \\
d^{\prime} \text { argumentaires pro- et } \\
\text { anti- autour d'un sujet } \\
\text { polémique ayant une base } \\
\text { scientifique }\end{array}$ \\
\hline Pourquoi? & $\begin{array}{l}\text { Informations et } \\
\text { cadrage du projet }\end{array}$ & $\begin{array}{l}\text { Adoption de bonnes } \\
\text { méthodes pour la } \\
\text { bibliographie }\end{array}$ & $\begin{array}{l}\text { Accompagnement } \\
\text { formatif, compréhension } \\
\text { du sujet et validation des } \\
\text { documents }\end{array}$ & $\begin{array}{l}\text { Définir une stratégie de } \\
\text { communication } \\
\text { orale }\end{array}$ & $\begin{array}{l}\text { Autonomie, travail de } \\
\text { groupe, pratique } \\
\text { documentaire, synthèse, } \\
\text { restitution de qualité }\end{array}$ \\
\hline Avec quoi ? & $\begin{array}{l}\text { Réunion de } \\
\text { lancement }\end{array}$ & $\begin{array}{l}2 \text { séances sur la recherche } \\
\text { bibliographique, plagiat et } \\
\text { citation }\end{array}$ & $\begin{array}{l}4 \text { séances de supervision } \\
\text { avec } 1 \text { groupe } \\
\text { d'étudiants }\end{array}$ & $\begin{array}{l}2 \text { séances techniques de } \\
\text { communication et } \\
\text { argumentaire }\end{array}$ & $\begin{array}{l}\text { Interactions équipe } \\
\text { enseignante - étudiants }\end{array}$ \\
\hline Où? & Salle de cours & $\begin{array}{l}\text { BU - salle de formation } \\
\text { (postes informatiques) }\end{array}$ & Laboratoire de recherche & Salle de cours & $\begin{array}{l}\text { Domicile - espaces de } \\
\text { travail à l'Université }\end{array}$ \\
\hline
\end{tabular}

9 La première séance permet au coordonnateur de présenter à la vingtaine d'étudiants l'unité d'enseignement, ses objectifs, ses contenus et les modalités d'évaluation. Mais c'est surtout l'occasion de contractualiser oralement l'engagement de chaque membre de l'équipe pédagogique ainsi que celui des étudiants. Les documents utilisés comme supports de ces informations sont ensuite diffusés aux étudiants et à l'équipe pédagogique.

La bibliothécaire accueille les étudiants pour deux séances. La première amène les étudiants à définir les contours de leur sujet et les concepts/mots-clefs s'y référant, puis à repérer et interroger de manière efficace diverses sources d'information (catalogues, bases de données, sites web fiables...) utiles à leur recherche documentaire. La seconde consiste en une sensibilisation au plagiat et à un apprentissage de la pratique de citation des sources. Durant ces séances, une large part est faite à la découverte, aux exercices, aux questions. Celles-ci portent sur le type d'information/de document pertinent pour le sujet à traiter, le/les auteur(s) d'un site web, la présentation $d u$ travail à rendre, les documents à citer dans les références bibliographiques, etc. C'est aussi l'occasion pour les étudiants d'avoir un premier temps de travail en contact avec les autres membres de leur groupe, tout en étant accompagnés dès le début de leur travail de recherche d'information.

Le tuteur veille à ce que les étudiants comprennent le sujet, qu'ils ne se perdent pas dans trop d'informations. Il juge de la pertinence et de la synthèse des informations extraites des documents. Ces étapes se réalisent soit par des séances présentielles pendant lesquelles les étudiants, souvent quatre (un minimum de deux étant obligatoire), font état de leurs avancées et échangent avec le tuteur. Ce peut être l'occasion de réguler si nécessaire la vie et le travail du groupe. Les étudiants doivent rendre compte d'une progression de leurs travaux, les rencontres peuvent être complétées par des échanges à distance. Le tuteur aide à la sélection et à la synthèse des documents les plus pertinents pour que ces derniers apparaissent au niveau du support de présentation orale. Après cette présentation orale, il rencontre encore une fois les étudiants pour dresser un bilan de l'AMS. 
L'enseignante de sciences de l'éducation intervient à deux reprises. Il s'agit d'abord d'apprendre à organiser les différents arguments pour obtenir un argumentaire structuré, cohérent. C'est aussi l'occasion de revoir à l'université le vocabulaire lié à l'argumentation. La première intervention reprend les résultats des recherches et des discussions avec le tuteur et la bibliothécaire, pour distinguer faits, argument d'autorité, conclusions d'études, arguments découlant d'un raisonnement causeconséquence, arguments subjectifs. La seconde séance est consacrée à la préparation de la prestation orale, la gestion du temps, la répartition de la parole entre les membres, les réponses aux questions. La grille utilisée pour l'évaluation (cf. Tableau 2), élaborée par l'équipe pédagogique, sert de référence aux étudiants pour auto-évaluer leur préparation en amont de la présentation orale. Ce document est important dans l'accompagnement car il indique dès le début de l'activité (il est distribué lors de la première séance) les champs pour lesquels les étudiants sont accompagnés, la recherche d'informations, la construction d'un contenu argumenté, la présentation orale. Il est utilisé tout au long du processus d'accompagnement.

Tableau 2. Grille d'évaluation élaborée par l'équipe pédagogique

\begin{tabular}{|c|c|c|}
\hline & & Barème \\
\hline \multirow{7}{*}{$\begin{array}{l}\text { Présentation } \\
\text { orale }\end{array}$} & 1. Structuration du propos & 2 \\
\hline & Le discours est construit et précis : annonce d'un plan, transitions entre les différentes parties de l'exposé, conclusion & \\
\hline & 2. Maîtrise de la langue & 2 \\
\hline & Qualité du vocabulaire et de la syntaxe - Bon usage du vocabulaire scientifique & \\
\hline & 3. Qualité des diapositives & 2 \\
\hline & Les diapositives sont bien construites, pertinentes par rapport à l'exposé, les illustrations aident à la compréhension & \\
\hline & $\begin{array}{l}\text { 4. Capacité à intéresser l'auditoire } \\
\text { Distance par rapport aux notes préparées - Les gestes et le regard renforcent l'intérêt }\end{array}$ & 1 \\
\hline \multirow{5}{*}{$\begin{array}{l}\text { Contenu de } \\
\text { l'exposé }\end{array}$} & $\begin{array}{l}\text { 1. Contextualisation du sujet } \\
\text { Présentation du sujet selon le contexte des sources }\end{array}$ & 2 \\
\hline & 2. Capacité à adopter une posture réflexive & 2 \\
\hline & Positionnement des étudiants vis-à-vis du sujet ... & \\
\hline & $\begin{array}{l}\text { 3. Réponses aux questions } \\
\text { Capacité à entrer en dialogue avec le jury : les réponses sont claires et argumentées }\end{array}$ & 2 \\
\hline & $\begin{array}{l}\text { 4. Répartition du travail } \\
\text { Répartition du temps de parole lors des présentations et de la discussion avec le jury }\end{array}$ & 2 \\
\hline \multirow{2}{*}{ Bibliographie } & $\begin{array}{l}\text { 1. Pertinence des documents cités } \\
\text { Les documents sont variés, adaptés au sujet et fiables }\end{array}$ & 1,5 \\
\hline & $\begin{array}{l}\text { 2. Citation des documents } \\
\text { Les documents sont cités selon la norme (citation dans la diapo + bibliographie finale) }\end{array}$ & 3,5 \\
\hline
\end{tabular}

13 La pédagogie choisie pour cette AMS a pour ambition d'assurer une fonction sociale de constitution $\mathrm{du}$ groupe, une fonction pédagogique en développant de nouveaux apprentissages et une fonction de développement personnel en favorisant l'ouverture vers des sujets sociétaux. Dans un premier temps, nous nous interrogeons sur les effets de l'accompagnement chez les étudiants. Les modalités de travail mises en place, notamment le travail de groupe en présentiel et majoritairement hors présentiel, influent-t-elle sur la construction du groupe, sa cohésion? Les étudiants perçoivent-ils les intentions pédagogiques de cette AMS? Quelle place attribuent-ils au groupe dans leur développement de compétences personnelles? Pour lesquelles? Les sujets proposés (cf. liste en annexe), reflet de questions actuelles dans la société, contribuent-ils au développement personnel de chacun?

14 Il convient également d'examiner l'hypothèse d'effets produits sur l'équipe qui prend en charge le dispositif présenté dans cet article. 


\subsection{L'accompagnement par une équipe pluridisciplinaire} qui a aussi pour mission, en plus de l'acquisition de documentation, celle d'accompagner les usagers à son utilisation. Les bibliothécaires sont amenés à organiser et à animer des formations aux " compétences informationnelles ", c'est le rôle du «bibliothécaire-formateur » (Puaud, 2017). Dans cette optique il semble que la qualité des enseignements et des ressources organisées par les bibliothèques et visant au développement des compétences informationnelles soit conditionnée par une réelle collaboration interprofessionnelle enseignants-bibliothécaires (Bélanger et al., 2017). Et plus généralement, il s'agit de l'enjeu d'une articulation, une collaboration, un décloisonnement entre les équipes pédagogiques et les services de documentation dans le contexte de la transformation pédagogique de l'enseignement supérieur (Paivandi, 2016). Pourtant la place des personnels de bibliothèque dans les cursus de formations universitaires et les équipes pédagogiques n'est pas toujours évidente, et on constate souvent plus une juxtaposition qu'une véritable intégration dans les équipes. À l'université de Lorraine, tous les étudiants de licence biologie bénéficient, dans le cadre des cours de méthodologie, d'heures de découverte de la bibliothèque et de recherche de documents. réalités (Fave-Bonnet, 2003), professeur, maitre de conférences, assistant, vacataire, etc. Plusieurs articles cités par de Hosson et al. (2015) indiquent qu'eu égard à leur formation qui privilégie les connaissances et la socialisation disciplinaire, les enseignants-chercheurs restent attachés à leur discipline. Au sein de l'AMS, le tuteur (chercheur ou enseignant-chercheur) vérifie l'argumentaire scientifique, ensemble des arguments qui viennent à l'appui d'une opinion ${ }^{4}$ préparé en amont par la recherche d'informations. Cependant, dans la plupart des cas, il n'est pas dans son domaine de spécialité. Les thématiques abordées relèvent davantage de préoccupations sociétales que des domaines d'expertise des auteurs des questions posées. Par exemple le sujet «Les comprimés homéopathiques, des fake news comme les autres? » est proposé par un chargé de recherche en biologie cellulaire, et le tuteur du sujet « la fusion nucléaire, la vraie solution énergétique » est maître de conférences en biochimie (voir aussi en annexe le tableau complet récapitulant tous les sujets proposés et leurs auteurs). Comment chaque enseignant vit-il cet "écart " par rapport au champ dans lequel il intervient? De plus Cosnefroy et Annoot (2014) remarquent que la formation universitaire s'éloigne de plus en plus de l'enseignement magistral pour aller vers une posture d'accompagnement par l'enseignant du supérieur. Ils ajoutent que cette posture d'accompagnant est mal définie mais un consensus se dégage sur le fait qu'elle gomme la relation hiérarchique entre l'enseignant et l'apprenant, qu'elle suggère un autre rapport à l'expertise et s'oppose au guidage dans lequel le guide connaît le but à atteindre et trace le chemin de l'apprenant (Ciekanski, 2011, p.14). Le terme " posture » présente l'avantage selon Lameul (2005) de faire référence à la fois à « un positionnement dans l'espace » et « est l'expression d'un état mental, façonnée par nos croyances et orientée par nos intentions qui exerce une influence directrice et dynamique sur nos actions, en leur donnant sens et justification» (p. 5). Plusieurs études soulignent que les pratiques sont dépendantes du choix des enseignants (Tremblay-Wragg et al., 2018), et qu'elles sont bien souvent magistro-centrées (Demougeot-Lebel, 2014; Kiffer, 2018). Mais l'enseignant universitaire peut être amené 
à occuper un rôle de tuteur dans le cadre d'activités pédagogiques pédo-centrées, en apportant une aide individualisée et jouant le rôle de facilitateur dans la réussite et le développement des tutorés (Barbier, 1996). Notre hypothèse de travail est que le fait de participer à des enseignements pédo-centrés dans cette AMS a des répercussions sur la posture globale de l'enseignant-chercheur qui pourrait être amené à diversifier ses stratégies pédagogiques en utilisant des principes pédagogiques qui permettent aux étudiants d'être actifs, facteur de réussite pour les étudiants (Freeman et al., 2014). Les enseignants engagés ont-ils conscience d'un changement de posture dans leur pratique professionnelle?

17 L'équipe d'enseignants intervenant en CMI est composée de spécialistes soit de sciences biologiques soit de sciences humaines. Elle souhaite faire évoluer le cloisonnement disciplinaire, favoriser les relations avec le spécialiste de l'information et ainsi constituer une équipe pluridisciplinaire. Celle-ci peut être vue pour Delattre (1977, cité dans Rege Colet, 1993) comme un groupe au sein duquel «la pluridisciplinarité est l'association des disciplines qui concourent à une réalisation commune, mais sans que chaque discipline ait à modifier sensiblement sa propre vision des choses et ses propres méthodes » (p. 21). Sauf lors de l'évaluation, les interventions ne sont pas conjointes, au sens où deux spécialistes de disciplines différentes interviennent dans une même séance sur un même contenu.

Plusieurs outils méthodologiques sont convoqués afin d'apporter des éléments de réponse aux questions qui viennent d'être formulées dans ce paragraphe.

\section{Méthodologie}

Deux corpus de données sont constitués. Le premier rassemble des informations déjà disponibles, liées aux traces résultant des mises en œuvre successives de l'AMS, le second est créé pour questionner les membres de l'équipe pédagogique.

Concernant les étudiants, nous disposons de différents documents utilisés pour l'évaluation. Tout d'abord ont été archivés les résultats de l'évaluation sommative, laquelle porte sur le diaporama utilisé lors du débat, la prestation orale et la bibliographie. La bibliographie, initialement faiblement prise en compte, est depuis trois ans intégrée de manière plus formelle dans la grille de notation afin de montrer aux étudiants l'importance de l'appui sur la documentation pour ce projet. La bibliothécaire note la qualité des sources citées et leur présentation. Ensuite sont aussi conservés les questionnaires individuels d'autoévaluation, que les étudiants renvoient par mail au coordonnateur après la présentation orale (les questions posées sont rappelées dans la présentation des résultats, cf. tableaux 3, 4 et 5 dans la partie "Résultats»). Ces questionnaires ont été introduits dès 2016 afin d'amener les étudiants à une réflexion sur leur travail et leur vécu du projet à travers le travail de groupe. Ce travail de réflexivité est obligatoire, il fait partie de l'évaluation. De ce fait la totalité des questionnaires administrés a pu être analysée, soit 54 questionnaires : 10 en 2016-2017, 13 en 2017-2018, 19 en 2018-2019, 12 en 2019-2020. Les questionnaires sont ensuite anonymisés avant d'être diffusés à l'équipe pédagogique qui fait ainsi évoluer le dispositif. Les réponses à ces questions ouvertes ont été analysées et organisées en items présentés dans les tableaux de la partie résultats.

21 Si les enseignants avaient conscience de fonctionner dans un registre différent de leurs habitudes, des discussions informelles attestaient des changements opérés mais 
l'écriture de cet article a été l'occasion d'objectiver les évolutions professionnelles. Un questionnaire à destination des tuteurs a été élaboré par les auteurs en se basant sur les dimensions impliquées dans la définition de la posture selon Lameul (2005): expérience, biographie, croyance, intention et action. Des questions sur l'évolution des pratiques pédagogiques dans l'ensemble de leurs enseignements avant et après leur participation en tant que tuteur dans cette AMS ont aussi été posées (cf. guide d'entretien en annexe). L'échantillon des tuteurs mobilisés pour répondre à ce questionnaire à travers des entretiens semi-directifs est constitué d'enseignantschercheurs ( $\mathrm{PR}$ et $\mathrm{MCF}$ ) et d'un chercheur (CR) ayant fait partie de l'équipe pédagogique de cette AMS plus d'une fois ${ }^{5}$. Ainsi le critère de participation à de multiples reprises permet aux tuteurs d'avoir une réflexion sur leur pratique et son évolution potentielle. Notons que sur cette base ce sont six tuteurs (avec une participation de trois à cinq fois à cette AMS) qui ont été sollicités pour des entretiens semi directifs. Le seul tuteur qui n'a pas donné suite à la demande d'entretien a quitté l'équipe pédagogique depuis deux ans. La totalité des tuteurs actuels s'est prêtée à l'exercice.

\section{Résultats}

22 Autant du côté des accompagnateurs (équipe pédagogique) que des accompagnés (étudiants) l'analyse des résultats fournit des indicateurs sur les effets du dispositif mis en place par l'équipe pluridisciplinaire pour accompagner dans l'appropriation de la littéracie universitaire.

\subsection{Pour les étudiants}

23 Le premier descripteur concerne la qualité des productions. Chaque année, les notes obtenues sont satisfaisantes et au-dessus de la moyenne (13,83/20 en n-3, 15,23 en n-2, 16,73 en n-1), plaçant donc les étudiants en situation de réussite, un des objectifs de l'AMS. Qualitativement, les bibliographies présentées sont de bonne qualité pour tous les groupes. Même si cette information n'est pas documentée ici, il convient de préciser que les compétences acquises dans la recherche et l'appropriation des différents écrits universitaires sont pointées en seconde année par les encadrants de l'atelier en laboratoire. Dans cette autre AMS, les étudiants doivent s'approprier un sujet de recherche grâce à la lecture d'un écrit de recherche fourni par le chercheur référent de l'atelier puis rechercher et comprendre d'autres articles scientifiques. Tout au long du cursus, la progressivité prévue dans la maquette de formation pour le développement de compétences dans le champ de la littéracie scientifique semble être efficace. Les rapports de tuteur de stage et d'atelier de laboratoire ainsi que les mémoires rédigés en master en témoignent. Ces différents éléments, repérés en première année puis durant le cursus, indiquent donc un développement de compétences informationnelles et de littéracie scientifique initié dès la première AMS.

Présentées dans les tableaux suivants (cf. tableaux 3, 4 et 5), les réponses données dans le questionnaire d'auto-évaluation distribué à la fin de l'AMS, apportent des informations sur l'influence des modalités de travail, notamment du travail en groupe. Ainsi un second descripteur des effets de l'accompagnement concerne sa fonction sociale. Le nombre d'heures de travail fourni (cf. Tableau 3) indique une implication 
forte des étudiants dans le travail demandé, avec un volume moyen horaire de travail en groupe supérieur au volume moyen de travail individuel. Cet investissement est sans doute à rapprocher de la compréhension de l'AMS par les étudiants. Ils reconnaissent majoritairement (43 réponses sur 54 ) le travail d'équipe comme un des objectifs de cette unité d'enseignement (cf. tableau 4).

Tableau 3. Réponses des étudiants sur le nombre d'heures travaillées sur le projet

\begin{tabular}{|l|l|l|l|l|l|l|}
\hline Question posée & \multicolumn{5}{|l|}{ Répartition des réponses des étudiants (n=50) } \\
\hline & $<5 \mathrm{~h}$ & $\begin{array}{l}\text { Entre } 5 \text { et } \\
10 \mathrm{~h}\end{array}$ & $\begin{array}{l}\text { Entre 11 et } \\
15 \mathrm{~h}\end{array}$ & $\begin{array}{l}\text { Entre 16 et } \\
20 \mathrm{~h}\end{array}$ & $>20 \mathrm{~h}$ & $\begin{array}{l}\text { Moyennes } \\
\text { heures }\end{array}$ \\
\hline $\begin{array}{l}\text { Combien d'heures ai-je travaillé } \\
\text { sur ce projet seul? }\end{array}$ & 3 & 30 & 11 & 2 & 4 & 10,5 \\
\hline $\begin{array}{l}\text { Combien d'heures ai-je travaillé } \\
\text { sur ce projet en groupe? }\end{array}$ & 1 & 14 & 22 & 8 & 4 & 13,6 \\
\hline
\end{tabular}

Pour autant, dans leur ressenti vis à vis du travail en groupe (cf. tableau 5), les éléments de réponses regroupés sous l'item "efficacité, satisfaction » (22 réponses sur 54 ) sont un peu plus nombreux que ceux regroupés sous l'item "affinités, entente, confiance " (19 réponses sur 54 ) et très peu indiquent le travail en groupe comme moyen pour faire connaissance en début d'année (4). Cette dernière réponse s'explique peut-être par l'adhésion de tous les étudiants dès la première année à l'association des étudiants CMI, laquelle organise des évènements extra-universitaires et dispose d'un local où les étudiants CMI se retrouvent souvent.

Ainsi la fonction sociale du dispositif semble moins importante que la fonction pédagogique, troisième descripteur des effets du dispositif. Les étudiants citent, après le travail d'équipe, comme objectifs de cette unité d'enseignement, la recherche d'information (cf. Tableau 4) suivi de près par la présentation orale (environ la moitié des réponses pour chaque item). Ces données sont en cohérence avec la présentation en début d'année par le coordonnateur de l'AMS, montrant la bonne compréhension des visées par les étudiants. Cependant, associés à la recherche d'information, les étudiants ne sont pas aussi nombreux à choisir comme objectifs les autres items liés à l'information (extraction, vérification, citation). Interrogés oralement sur cette différence, ils répondent que pour eux ces précisions sont intégrées dans une recherche d'information de qualité. Ils semblent donc considérer un processus de recherche d'information (comprenant la collecte, la sélection, la vérification, l'organisation de la restitution et le référencement) plutôt qu'une opération de recherche.

Tableau 4. Analyse par item du contenu des réponses des étudiants sur leur compréhension des objectifs de l'UE

\begin{tabular}{|l|l|l|}
\hline Question posée & Répartition des éléments de réponse $(\mathrm{n}=54)$ \\
\hline $\begin{array}{l}\text { A mon avis, quels étaient les } \\
\text { objectifs de cette UE? }\end{array}$ & Travail d'équipe & 43 \\
\cline { 2 - 3 }
\end{tabular}




\begin{tabular}{|l|c|}
\hline Recherche d'information & 28 \\
\hline $\begin{array}{l}\text { Présentation d'un oral devant le jury, diaporama, } \\
\text { argumentation }\end{array}$ & 24 \\
\hline Organisation du travail, planification & 16 \\
\hline Découverte d'un sujet, de l'actualité scientifique & 16 \\
\hline Vérification de la qualité de l'information & 15 \\
\hline $\begin{array}{l}\text { Extraction de l'information, analyse, synthèse, } \\
\text { organisation de l'information }\end{array}$ & 14 \\
\hline Adoption d'une démarche scientifique & 13 \\
\hline $\begin{array}{l}\text { Citation des sources, rédaction des références } \\
\text { bibliographiques }\end{array}$ & 11 \\
\hline
\end{tabular}

Si les réponses sur le vécu (cf. Tableau 5) confirment l'intérêt du travail d'équipe (partage des tâches, compréhension du sujet), le groupe paraît moins nécessaire pour la préparation de l'oral. Malgré l'importance de cette phase de l'AMS, reconnue par les étudiants, cet item est toujours le moins cité, tant dans le rôle pour lequel l'étudiant se sent moteur que ce qu'il apprend du groupe. Cette réponse est un peu surprenante si on précise que la forme de la présentation (tirage au sort le jour même du rôle dans la présentation) rend nécessaire la cohésion du groupe et une préparation commune.

Les réponses indiquent aussi l'importance du groupe pour l'entrée dans la littéracie, à la fois dans le rassemblement d'idées, la compréhension du sujet à partir des documents sélectionnés mais aussi pour la rédaction d'un argumentaire scientifique (ce sont les deux premiers items sélectionnés). Le groupe semble donc contribuer au développement des compétences personnelles.

Tableau 5. Analyse par item des réponses des étudiants sur leur vécu du projet de groupe

\begin{tabular}{|c|c|c|}
\hline Question posée & Répartition des éléments de réponse $(n=54)$ & \\
\hline \multirow{5}{*}{$\begin{array}{l}\text { Quel est mon ressenti vis-à-vis du } \\
\text { travail en groupe? }\end{array}$} & $\begin{array}{l}\text { Organisation du travail, partage des tâches, } \\
\text { complémentarité }\end{array}$ & 34 \\
\hline & Efficacité, satisfaction & 22 \\
\hline & Affinités, entente, confiance & 19 \\
\hline & Débat, confrontation d'idées, écoute & 18 \\
\hline & Faire connaissance en début d'année universitaire & 4 \\
\hline Sans le groupe, j'aurais eu du mal & Rassemblement d'idées, débat, compréhension du sujet & 31 \\
\hline
\end{tabular}




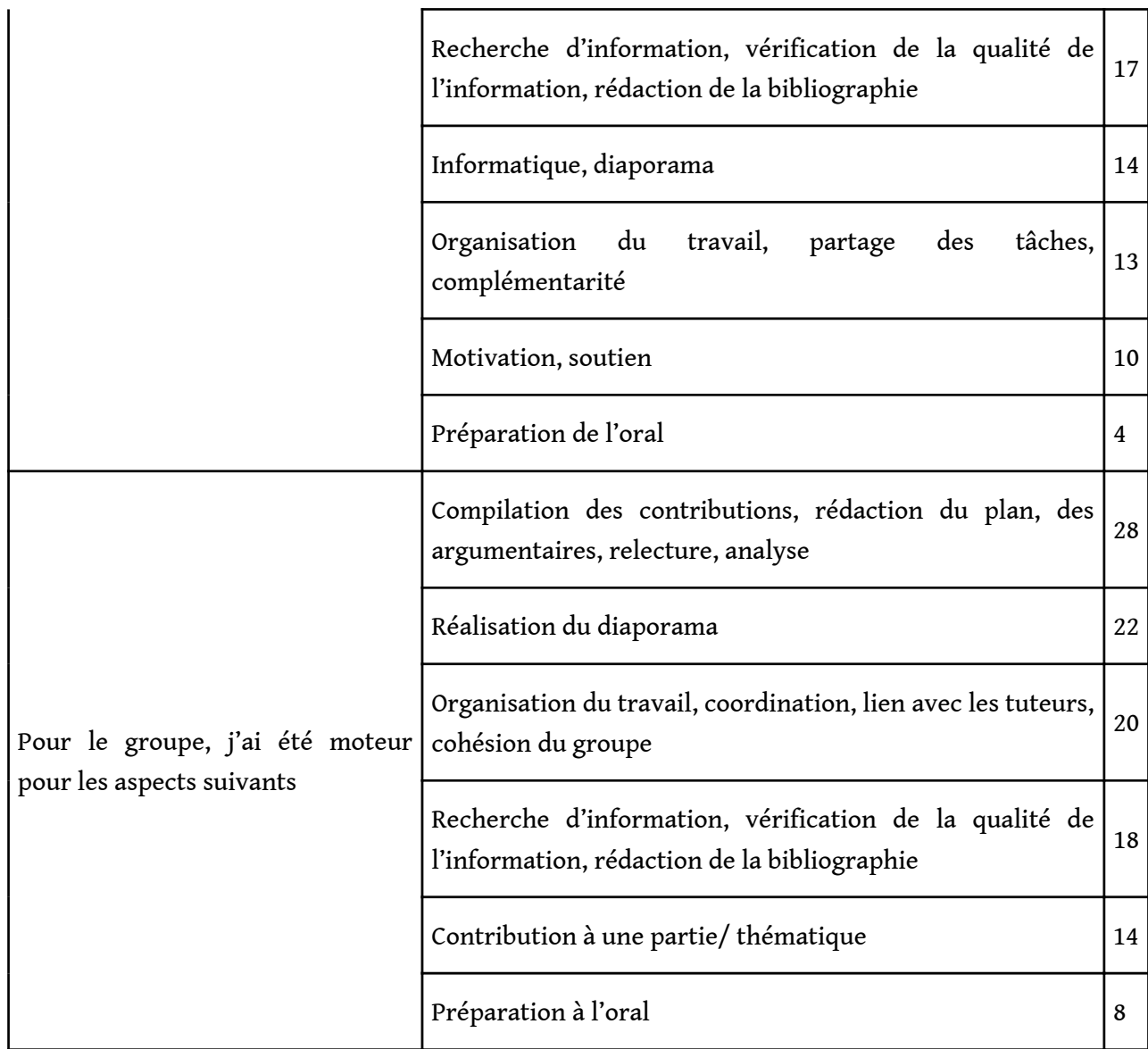

Enfin, une question a été ajoutée depuis l'année universitaire 2018-2019) pour comprendre le lien qu'établissent les étudiants entre le sujet proposé et la spécialité du tuteur (cf. Tableau 6). Alors que les groupes rencontrent les tuteurs plusieurs fois, la plupart du temps dans leur laboratoire, que certains interviennent en cours, le sujet proposé apparaît majoritairement comme lié au champ d'expertise du tuteur alors que l'annexe 1 témoigne surtout de l'intérêt personnel ou d'actualité du thème comme critère de choix. Très peu ( 5 sur 31 réponses) perçoivent le sujet comme relevant d'une actualité scientifique. En ce sens, les réponses des étudiants indiquent qu'une des fonctions du dispositif (contribuer au développement personnel en renforçant sa culture générale) n'est pas encore assurée.

Tableau 6. Analyse par item sur le lien entre sujet et profession du tuteur

\begin{tabular}{|l|l|l|}
\hline \multirow{2}{*}{ Question posée } & $\begin{array}{l}\text { Répartition des éléments de } \\
\text { réponse }(\mathrm{n}=31)\end{array}$ \\
\hline \multirow{2}{*}{$\begin{array}{l}\text { À votre avis, quel rapport y-a-t-il entre votre sujet et la } \\
\text { profession de votre tuteur? }\end{array}$} & $\begin{array}{l}\text { La thématique de recherche du } \\
\text { tuteur }\end{array}$ & 15 \\
\cline { 2 - 3 } & $\begin{array}{l}\text { La démarche scientifique } \\
\text { Curiosité, intérêt personnel, } \\
\text { hobby du tuteur }\end{array}$ & 7 \\
\cline { 2 - 3 }
\end{tabular}




$$
\begin{aligned}
& \begin{array}{l}
\text { C'est un sujet d'actualité } \\
\text { scientifique }
\end{array} \\
& 5
\end{aligned}
$$

30 L'analyse des résultats des évaluations sommatives et des réponses des étudiants aux questionnaires d'auto-évaluation montre donc que cette AMS assure prioritairement une fonction pédagogique de développement de compétences informationnelles, en lien avec une entrée dans la littéracie scientifique. La modalité de travail retenue, principalement le travail de groupe, est davantage repérée par les étudiants comme un mode efficient d'apprentissage qu'un moyen de socialisation en début de cursus universitaire. Le troisième effet recherché, développer la culture générale, ne semble pas encore installé et interroge les tuteurs.

\subsection{Pour les tuteurs}

L'enquête s'intéresse d'abord au profil des tuteurs, leur présence dans cette unité d'enseignement relevant d'un choix personnel. L'évolution de leurs croyances eu égard à l'accompagnement et le changement de posture généré par cette expérience d'enseignement apparaissent ensuite.

\subsubsection{Profils des tuteurs}

32 L'analyse de la partie biographique du questionnaire permet de caractériser les tuteurs. Ils avaient des expériences variables comme enseignant universitaire lorsqu'ils sont entrés dans l'équipe pédagogique de cette AMS : entre 30 heures et neuf années. Le tuteur ayant actuellement un statut de chargé de recherche au CNRS en biologie-santé fait état de plus de trois ans d'enseignement en demi-service notamment lors de la préparation de son doctorat. Leur parcours de formation initiale est très majoritairement universitaire (un seul tuteur a passé deux ans en BTS avant d'intégrer un cursus universitaire). Un seul mentionne un apprentissage par problème en groupe lors de sa deuxième année universitaire, mais avec une absence de tuteur. Les autres n'ont eu eux-mêmes soit, aucune expérience de pédagogie universitaire autre que magistro-centrée, soit des expériences de restitutions bibliographiques de groupe en master avec ou sans tuteur.

Deux des tuteurs en poste, depuis quatre (PR1) ou neuf ans (MCF1) au moment où ils intègrent l'équipe pédagogique, ont des pratiques pédagogiques exclusivement magistro-centrées. Parmi eux, le coordonnateur de cette AMS (PR1) avait suivi, six ans auparavant à l'université de Sherbrooke un microprogramme de second cycle en pédagogie post-secondaire, sans trouver l'opportunité de mettre en pratique des pédagogies pédo-centrées. Les autres tuteurs intègrent l'équipe pédagogique de l'AMS rapidement après leur prise de poste.

\subsubsection{Postures des tuteurs dans cette AMS}

On peut distinguer la situation des trois tuteurs qui étaient dans l'équipe pédagogique initiale (MCF1, MCF3, PR1) de celle des deux autres qui ont joué un rôle de tuteur alors que l'AMS existait depuis quatre ans (MCF2 et CR1). L'équipe de départ a construit son rôle de tuteur à travers différentes manières de faire, dont celle de tutorer ces groupes 
de première année avec un objectif de rendu trop proche des attentes d'étudiants de master, donc trop difficile à atteindre. Une autre approche était d'avoir une idée trop précise des arguments à développer et du comment trouver la littératie associée au sujet, ce qui entraînait un manque de distance du tuteur par rapport à son groupe et un résultat reflétant trop fortement l'opinion du tuteur.

La seconde situation est celle des tuteurs qui ont reçu une présentation "assez claire » (MCF2) du rôle de tuteur par le responsable pour lequel il s'agit de «transmettre une boite à outil [...] leur permettant de développer un esprit critique [...] être capable de voir quelles sources sont utilisables ou non » (PR1). La croyance, en tant qu'élément constitutif de la posture selon Lameul (2005), partagée par les deux catégories de tuteurs, lors de leur première participation à l'AMS était, dans une logique d'enseignement transmissif, de faire bénéficier les étudiants de leur expertise dans leur domaine de recherche et d'encadrer plus qu'accompagner. Or, donner un sujet « trop proche du sujet de recherche " (PR1 et MCF2) du tuteur et donc "trop complexe» (MCF2) et "trop élitiste" (PR1) semble poser un certain nombre de problèmes au regard du type d'accompagnement attendu.

Lors des séances avec les tuteurs, il arrive que des situations inédites se mettent en place. Ainsi, la pression du manque de disponibilité des uns et des autres peut faire que les tuteurs aient des déjeuners de travail avec leurs étudiants, ce qui constitue une posture différente. Cette forme de travail est peu habituelle, instituant un rapport enseignant-étudiant différent.

La posture adoptée actuellement par l'ensemble des tuteurs est «d'être source d'interrogations, ainsi si le groupe d'étudiants n'a pas réfléchi en amont le tuteur ne sert à rien » (MCF3) ou de donner sporadiquement des conseils ou de s'assurer que les jalons d'avancement du projet sont respectés : «jouer le rôle de maître du temps » (PR1).

Par ailleurs, les tuteurs font parfois des propositions de pistes d'argumentaire sans que les étudiants ne se les approprient. La littéracie utilisée est souvent bien différente de celle que le tuteur projetait dans le sujet qu'il proposait. Le tuteur doit aussi s'approprier les références et les sources d'information que les étudiants soumettent à son expertise et si besoin vérifier leur fiabilité, ce qui l'oblige parfois à s'écarter de sa zone de confort. De même, les médias utilisés varient, les étudiants privilégiant les ressources sur internet, notamment les sites web alors que les tuteurs sont davantage attachés aux articles de recherche. Ces différences de sources sont l'occasion de discussion entre les deux parties. L'angle choisi par les étudiants peut-être très différent de la façon dont le tuteur aurait développé le sujet et « on (le tuteur) ne peut pas construire l'argumentaire à leur place» (MCF1). Le tuteur doit alors accepter de sortir de son rôle d'expert et accompagner les groupes d'étudiants dans un projet concret qui ne lui appartient pas et ne peut pas être défini à l'avance.

Ainsi, après plusieurs années de participation à l'AMS, tous les tuteurs reconnaissent un changement de posture dans cette activité de mise en situation, notamment la mise à distance de leur spécialité disciplinaire. Les réponses au questionnaire indiquent aussi que le changement de posture est transféré dans d'autres unités d'enseignement à chaque fois que possible. Cependant le cadre de la maquette d'enseignement, accréditée pour cinq ans, constitue parfois un frein. 


\section{Discussion : un dispositif en perpétuelle évolution et ses répercussions} bonne intégration dans le cursus universitaire grâce au travail d'équipe et au suivi par les enseignants. Les résultats observés par les enseignants attestent de l'acquisition de compétences informationnelles et de lecture-compréhension des écrits universitaires dans le domaine de la biologie. En cela, l'AMS apparait comme un facteur de réussite des étudiants, tout en gardant en mémoire que ce dispositif s'adresse à une population étudiante peu à risque en termes d'échec et d'abandon. Il convient de signaler que $94 \%$ des étudiants en CMI valident leur première année de licence Sciences de la Vie avec $2,5 \%$ d'abandon, alors que $35 \%$ des étudiants du cursus «classique » valident leur première année avec un taux d'abandon de $17,5 \%$ des étudiants qui ne se présentent pas aux examens (données internes du département). L'enquête sur les effets du dispositif chez les étudiants reste à développer, notamment pour évaluer l'impact de la recherche et la production d'écrits d'argumentation sur l'empouvoirement des étudiants, leur capacité à agir sur des questions de société. Nous ne disposons pas non plus d'information sur comment les accompagnés vivent l'accompagnement, si ce dispositif change leur relation à l'enseignant et à l'équipe pédagogique, si ce rapport plus étroit entre accompagnant et accompagné favorise le développement des compétences.

41 Parallèlement, l'AMS a permis d'engager tous les enseignants-chercheurs impliqués dans un changement de posture professionnelle, en quittant leur position de « sachant » pour aller vers l'accompagnement. Tous montrent un niveau de satisfaction élevé de leur participation, qui «change de l'enseignement classique » (MCF1), modifie aussi la hiérarchie entre tuteur et étudiant, le rôle de l'enseignant, confirmant ainsi les articles de Cosnefroy et Annoot (2014) et Ciekanski (2011).

Enfin, il apparaît également que le fonctionnement en équipe pluridisciplinaire, comme le soulignent de Hosson et al. (2015), a favorisé le décloisonnement entre les disciplines. Il favorise également un transfert de la responsabilité sur tout le groupe et non sur l'enseignement-chercheur seul, apportant un enrichissement de l'activité.

\section{Perspectives}

Depuis sa mise en place cette AMS a évolué, prenant en compte les résultats des autoévaluations, mais aussi les discussions entre membres de l'équipe ou entre l'équipe et les étudiants. Ainsi, la place de la documentation a été revue, créant un nouveau rapport entre la bibliothécaire et les enseignants, propice au développement des compétences informationnelles. Cette évolution de la place de la bibliothécaire dans l'équipe pédagogique, les modifications de représentations entre enseignants chercheurs de sciences biologiques et de sciences humaines, ne sont pas examinées dans cet article. Il resterait aussi à examiner comment ce travail conjoint s'articule entre les différents membres de l'équipe et comment il est reçu par les étudiants.

On peut noter que les modifications introduites au cours des dernières années sont de moindre importance par rapport aux trois premières sessions. Le rôle des tuteurs est maintenant clairement défini grâce aux erreurs commises dans les premières années et

Revue internationale de pédagogie de l'enseignement supérieur, 36(2) | 2020 
aux échanges au sein de l'équipe pédagogique. Le dispositif constitue la première étape d'un processus d'autonomisation des étudiants dont la progressivité a été construite pour la maquette de formation (2018-2023) eu égard aux évolutions. C'est aussi la première phase de découverte de la littéracie universitaire, tout au long de leur cursus les étudiants s'approprient peu à peu les écrits scientifiques, leurs codes et leur structure, via des enseignements et lors d'activités de mise en situation, organisés de façon progressive. Ils ont ce rapport aux écrits à la fois en tant que lecteur (lecture rapide des caractéristiques et compréhension des idées principales) et en tant qu'auteur (rédaction d'un écrit et de sa bibliographie selon les exigences académiques). Il convient de préciser ici que l'unité d'enseignement décrite dans l'article n'est qu'un élément dans l'ensemble des AMS, engageant d'autres enseignants-chercheurs, personnels de documentation et d'orientation. Il semble donc nécessaire de créer maintenant des outils d'observation pour évaluer la construction des compétences informationnelles et l'appropriation de la littéracie universitaire.

Par ailleurs, depuis l'année universitaire 2018-2019, comme pour tous les projets de CMI, celui de découverte de l'argumentation scientifique fait l'objet d'une réflexion avec les personnels d'orientation. L'intégration des conseillères du service d'orientation et d'insertion professionnelle (SOIP) au sein de l'équipe permettrait d'accompagner les étudiants dans la mise en relation entre les activités vécues et la construction de leur projet personnel et professionnel.

Jusqu'à maintenant, le groupe d'intervenants est stable, les tuteurs variant un peu en fonction des sujets choisis par les étudiants. Tous les tuteurs ont remarqué la maturité des étudiants de CMI première année et la qualité du travail de groupe. Grâce à cette expérience positive, des modifications ou des initiatives ont été introduites dans les pratiques pédagogiques des enseignants-chercheurs, à chaque fois que possible. À l'exception du chargé de recherche qui n'a pas d'autre activité d'enseignement, tous utilisent dorénavant dans leurs enseignements de la pédagogie active comme caractère inhérent à cet accompagnement sans renoncer complètement au transmissif. Actuellement tous les tuteurs sont impliqués dans d'autres AMS, en deuxième ou troisième année à la faculté des sciences du cursus de licence classique ou CMI, ou en deuxième année de Licence de sciences pour la santé. Même si le pourcentage de tels dispositifs reste minoritaire dans les services des enseignants, il existe une multiplication des activités pédo-centrées. Là aussi, il pourrait être intéressant d'observer en détail l'extension de ces pratiques professionnelles dans d'autres formes que les AMS.

Toutefois, ce dispositif qui semble donner satisfaction à tous les acteurs (les étudiants citent les AMS comme les points d'intérêt du CMI lors de leurs discussions avec des lycéens dans des portes ouvertes ou salon de l'orientation), n'est peut-être pas généralisable facilement pour des questions de coût et d'effectifs. Il constitue cependant une opportunité pour assurer un accompagnement à la rupture avec le lycée et une évolution globale des pratiques pédagogiques à l'université. 


\section{BIBLIOGRAPHY}

American Library Association (1989). Presidential Committee on Information Literacy : Final Report. http://www.ala.org/acrl/publications/whitepapers/presidential

Barbier, J.-M. (1996). Tutorat et fonction tutorale : quelques entrées d'analyse. Recherche \& formation, 22, 7-19. https://www.persee.fr/doc/refor_0988-1824_1996_num_22_1_1341

Bélanger, G., Boisvert, D., Lemieux, M.-M. et Séguin, C. (2017). Qualité des pratiques de développement des compétences informationnelles au sein du réseau de l'Université du Québec. Université du Québec [rapport]. http://rapport-qualite-pdci.uquebec.ca/Documents/Rapport-FODAR-

CI-2017.pdf

Chartrand, S.-G. et Elghazi, L. (2014). L'argumentation-1. Qu'est-ce qu'argumenter? Ecriture, 19(3). http://correspo.ccdmd.qc.ca/index.php/document/heureux-dun-printemps/largumentation-iquest-ce-quargumenter1/

Ciekanski, M. (2011). L'analyse ergonomique du travail d'accompagnement du conseiller dans les systèmes d'apprentissage autodirigé. Mélanges CRAPEL, 32, 10-23. http://www.atilf.fr/IMG/pdf/ 9-24_ciekanski.pdf

Cosnefroy, L. et Jézégou, A. (2013). Les processus d'autorégulation collective et individuelle au cours d'un apprentissage par projet. RIPES, 29(2). http://ripes.revues.org/744

Cosnefroy, L. et Annoot, E. (2014). Pourquoi s'intéresser à la posture d'accompagnement dans l'enseignement supérieur aujourd'hui ? Recherche \& formation, 77, 9-15. http:// journals.openedition.org/rechercheformation/2296

de Hosson, C., Décamp, N., Morand, E. et Robert, A. (2015). Approcher l'identité professionnelle d'enseignants universitaires de physique : un levier pour initier des changements de pratiques pédagogiques. RDST, 11, 161-196. https://journals.openedition.org/rdst/1014

Delcambre, I. et Lahanier-Reuter, D. (2012). Littéracies universitaires : présentation. Pratiques, 153-154, 3-19. http://journals.openedition.org/pratiques/1905

Demougeot-Lebel, J. (2014). Enseignants-chercheurs de la Génération Y : incidence sur les pratiques pédagogiques? RIPES, 30(3). http://journals.openedition.org/ripes/883

Fave-Bonnet, M.-F. (2003). Les universitaires : une identité professionnelle incertaine. Hermès, La Revue, 35(1), 195-202. https://www.cairn.info/revue-hermes-la-revue-2003-1-page-195.htm

Freeman, S., Eddy, S. L., McDonough, M., Smith, M. K., Okoroafor, N., Jordt, H. et Wenderoth, M. P. (2014). Active Learning increases student performance in Science, Engineering, and Mathematics. PNAS, 111(23), 8410-8415. https://doi.org/10.1073/pnas.1319030111

Garrau, M. (2018). Refuser la loi ORE. Multitudes, 71(2), 14-20. https://doi.org/10.3917/mult. 071.0014

Gettliffe, N. (2018). Accompagner l'acculturation aux écrits universitaires : les cours de méthodologie du travail universitaire. RIPES, 34(1). https://journals.openedition.org/ripes/1267

Houart, M., Baillet, D., Cobut, B., Vanden Eynde, C., Duchâteau, D., Goemaere, S., ...et Salmon, D. (2011). Focus sur les déclencheurs de l'évolution des actions d'accompagnement pédagogique. RIPES, 27(1). http://ripes.revues.org/464 
Jacquet, E., Gaume B., Gaillard Y., Descourvières E., Marangé P., Aigle B., ... et Gallon, C. (2014). Pédagogie renouvelée pour des cursus d'ingénierie à l'université. Colloque sur l'Enseignement des Technologies et des Sciences de l'Information et des Systèmes, CETSIS 2014, Besançon, France. https://hal.archives-ouvertes.fr/hal-01094950/document

Kiffer, S. (2018). Apprendre à enseigner dans le supérieur : quels modèles pour la construction des compétences des universitaires novices? RIPES, 34(3). http://journals.openedition.org/ripes/ 1654

Lameul, G. (2005). Quels effets de la médiatisation de la relation pédagogique sur la construction des postures professionnelles enseignantes? http://sif2005.mshparisnord.org/pdf/Lameul.pdf

Langevin, F. (2017) Bulletin de veille : Impact des compétences informationnelles (CI) sur la réussite étudiante. Tribune Compétences Informationnelles. https://tribuneci.wordpress.com/ 2017/01/10/veille-informationnelle-impact-des-competences-informationnelles-ci-sur-lareussite-etudiante

Lemieux, C. (2007). A quoi sert l'analyse des controverses. Mil neuf cent, revue d'histoire intellectuelle, 25. https://www.cairn.info/revue-mil-neuf-cent-2007-1-page-191.htm

Paivandi, S. (dir.) (2016). Articulation et collaboration entre les équipes pédagogiques et les services de documentation au cœur de la transformation pédagogique de l'enseignement supérieur [Étude réalisée par le LISEC, Laboratoire Interuniversitaire des Sciences de l'éducation et de la communication]. http://media.sup-numerique.gouv.fr/file/Publications/25/0/Rapport_LISEC_681250.pdf

Perret, C. (2014). Retour d'expérience d'une première évaluation du Plan Réussite en Licence à l'Université de Bourgogne : les leçons d'un difficile recueil des points de vue enseignants et étudiants. RIPES, 30(2). http://ripes.revues.org/816

Puaud, M. (2017). Portrait métier \#4 : Bibliothécaire-formatrice. BBF, 13, 92-94. http:// bbf.enssib.fr/consulter/bbf-2017-13-0092-011

Rege Colet, N. (1993). Pluridisciplinarité, interdisciplinarité, transdisciplinarité quelles perspectives en éducation? : étude du discours des professeurs de la Section des sciences de l'éducation de l'Université de Genève. Faculté de psychologie et des sciences de l'éducation, Université de Genève, Genève, Suisse.

Tremblay-Wragg, E. Raby, C. et Ménard, L. (2018). En quoi la diversité des stratégies pédagogiques participe-t-elle à la motivation à apprendre des étudiants? Etude d'un cas particulier. RIPES, 34(1). http://journals.openedition.org/ripes/1288

\section{NOTES}

1. http://reseau-figure.fr/wp-content/uploads/sites/121/2016/08/Referentiel-CMI-FIGURE_2016-2017.pdf

2. D'après les définitions proposées par le Centre National de Ressources Textuelles et Lexicales (https://cnrtl.fr/definition/accompagner)

3. http://cache.media.enseignementsup-recherche.gouv.fr/file/RERS_2018/83/2/depp-2018RERS-web_986832.pdf, page 189

4. En ligne : http://www.cnrtl.fr/definition/academie9/argumentaire

5. En effet, quatre tuteurs n'ont participé qu'une fois ce qui ne leur a pas donné l'opportunité de faire évoluer leurs pratiques au sein de cette AMS. Ce choix méthodologique évite le biais d'avoir travaillé avec un seul groupe d'étudiants, qui ne peut pas être représentatif de tous les groupes impliqués dans le dispositif. 


\section{ABSTRACTS}

Within the framework of a master's degree in Biological, Health and Environmental Engineering developed at the University of Lorraine in France, a multidisciplinary team offers first year students a Situational Awareness Activity (AMS). In groups of three or four, the students have to carry out scientific documentation research on a societal subject related to science and develop pro and con arguments that are then presented orally. The pedagogical team accompanies the students throughout the process, with the aim of fostering the integration and success of new students. It is also a question of reinforcing the skills built up in high school, in particular informational skills in order to facilitate the appropriation of university literacy. The article examines the effects of this support system on the students and their tutors. The analysis of the students' results, their answers to a self-evaluation survey and those of the tutors during semidirective interviews indicate that this AMS contributes to the acquisition of skills and the development of students' autonomy. The teachers report a change of stance in their role as tutors, a change that also transforms their professional practices in other courses.

Dans le cadre du Cursus Master Ingénieur (CMI) de Biologie Santé Environnement (BSE) développé à l'Université de Lorraine en France, une équipe pluridisciplinaire propose aux étudiants de première année de cette formation une Activité de Mise en Situation (AMS). Par groupe de trois ou quatre, ils/elles doivent effectuer une recherche de documentation scientifique sur un sujet de société lié aux sciences pour ensuite élaborer un argumentaire pro- et anti- qui sera présenté oralement. L'équipe pédagogique accompagne les étudiants tout au long $\mathrm{du}$ processus, dans l'objectif de favoriser l'intégration et la réussite des nouveaux étudiants. Il s'agit aussi de renforcer des compétences construites au lycée, notamment des compétences informationnelles afin de faciliter l'appropriation de la littéracie universitaire. L'article examine la question des effets de ce dispositif d'accompagnement sur les étudiants et les accompagnants. L'analyse des résultats des étudiants, de leurs réponses à une enquête d'auto-évaluation, de celles des tuteurs lors d'entretiens semi-directifs indique que cette AMS contribue à l'acquisition de compétences et au développement de l'autonomie des étudiants. Les enseignants témoignent d'un changement de posture dans leur rôle d'accompagnant, changement qui transforme aussi leurs pratiques professionnelles dans d'autres cours.

\section{INDEX}

Mots-clés: cursus master en ingénierie, littéracie scientifique, compétences informationnelles, équipe pluridisciplinaire

\section{AUTHORS}

\section{MARTINE PAINDORGE}

Institut national supérieur du professorat de l'éducation de Lorraine, martine.paindorge@univlorraine.fr

\section{VIRGINIE LANG}

Bibliothèque Universitaire des Sciences et Techniques, Direction de la Documentation et de l'Édition, Université de Lorraine, virginie.lang@univ-lorraine.fr 


\section{VIRGINIE LIBANTE}

Faculté des Sciences et Technologies, Université de Lorraine, virginie.libante@univ-lorraine.fr PASCAL REBOUL

Faculté de médecine, Université de Lorraine, pascal.reboul@univ-lorraine.fr

\section{BERTRAND AIGLE}

Faculté des Sciences et Technologies, Université de Lorraine, bertrand.aigle@univ-lorraine.fr 